\title{
In Situ Transmission Electron Microscopy Graphene Liquid Cell on Chemical Sodiation of Nickel Oxide Nanoparticle
}

Frank Jaksoni Mweta, ${ }^{1,2}$ Joon Ha Chang, ${ }^{1,2}$ Hyeon Kook Seo, ${ }^{1,2}$ Sung Joo Kim,,${ }^{1,2}$ Jun Young Cheong, ${ }^{1}$ II-Doo Kim, ${ }^{1 *}$ Jong Min Yook, ${ }^{1,2}$ and Jeong Yong Lee ${ }^{1,2}$.

${ }^{1 .}$ Department of Materials Science and Engineering, Korea Advanced Institute of Science \& Technology, Daejeon, Republic of Korea.

2. Center of Nanomaterials and Chemical Reactions, Institute for Basic Science (IBS), Daejeon, Republic of Korea.

Sodium-ion batteries (SIBs) are highly treated to be the complementary alternatives to lithium ion batteries (LIBs) with response to the abundance of cost-effective sodium material. Both sodium and lithium belong to the same main group, showing most similar chemical characteristics although Na has relatively large ionic radius compared to lithium, which limits its electrochemical performance [1]. For SIBs to fulfil its promise of being alternative to LIBs, there is a necessity to innovate effective and appropriate strategies to design various electrodes materials with functional structures [2]. Among the widely studied electrodes are the transition metal oxides (TMOs) as they exhibit high theoretical capacities for sodium storage via similar mechanisms to lithium [3].

Nickel oxide $(\mathrm{NiO})$ is one of the transition metal oxide been studied as effective electrode material for sodium ion batteries based on it is high theoretical specific capacity, good rate capability and excellent cycling performance for sodium storage. However, to date out of $718-\mathrm{mAh} \mathrm{g}^{-1}$ the sodiation theoretical capacity of $\mathrm{NiO}$, only $480 \mathrm{mAhg}^{-1}$ was practically achieved [4]. Almost $34 \%$ of this capacity is not usable. For efficiently utilizing this electrode material via suitable structure design, this study attempted to understand in deeply the chemical sodiation mechanism on $\mathrm{NiO}$ nanoparticles (NPs) through advanced in situ transmission electron microscopy approach using graphene liquid cell [5].

The experiments were conducted by including several steps such as (i) synthesis of monolayer graphene on $\mathrm{Cu}$ substrate using chemical vapor deposition (CVD), (ii) monolayer graphene transferred on the TEM grid (200 mesh Au grid), (iii) solution preparation; $0.5 \mathrm{mg}$ of $\mathrm{NiO}$ nanoparticles dissolved in $1 \mathrm{M}$ of $\mathrm{NaPF}_{6}$ liquid electrolyte consisting the solvent mixture of Ethylene carbonate, followed by entrapment of a liquid solution between layers of graphene [5]. During in situ TEM observations (Figure 1), under the impact of electron beam irradiation the sodium ions appeared to dissociate from $\mathrm{NaPF}_{6}$ while undergoes conversion reaction with $\mathrm{NiO} \mathrm{NPs}$ to form crystalline sodium oxide $\left(\mathrm{Na}_{2} \mathrm{O}\right)$ and Nickel nanoparticles (Ni NPs). The conversion reaction was investigated to be accompanied with several phenomena including coalescences, splitting and morphological change of the particle. Likewise, highresolution images (Figure 2) revealed the sodiation mechanisms to be accompanied with the formation of incoherent interphase boundaries between $\mathrm{Ni}$ and $\mathrm{Na}_{2} \mathrm{O}$ phases.

The incoherent interphase boundaries led to large lattice mismatch (highly atomic disordered) between the two phases. For advanced design of sodium ion battery with $\mathrm{NiO}$ electrode, effective control of the interfacial migration is recommended [6]. 
References:

[1] M. Sawick and L.L. Shwa, RSC Advances, 5 (2015), p.53129.

[2] L. D. Ellis et al, J.Electrochem.Soc.161(2014), A416-421

[3] W. Sun et al, Journal of power sources, 274(2015), p.755-761.

[4] K. He et al, Nano letters 15(2015), p.5755-5763.

[5] J. Yuk et al, Science 336(2012), pp.61-64.

[6] The authors acknowledge the funding from Korea CCS R\&D Center (KCRC) grant by the Korea government (Ministry of Science, ICT \& Future Planning) (No. NRF-2014M1A8A1049303), Wearable Platform Materials Technology Center (WMC) (NR-2016R1A5A1009926), and Institute for Basic Science (IBS) (IBS-R004-G3).
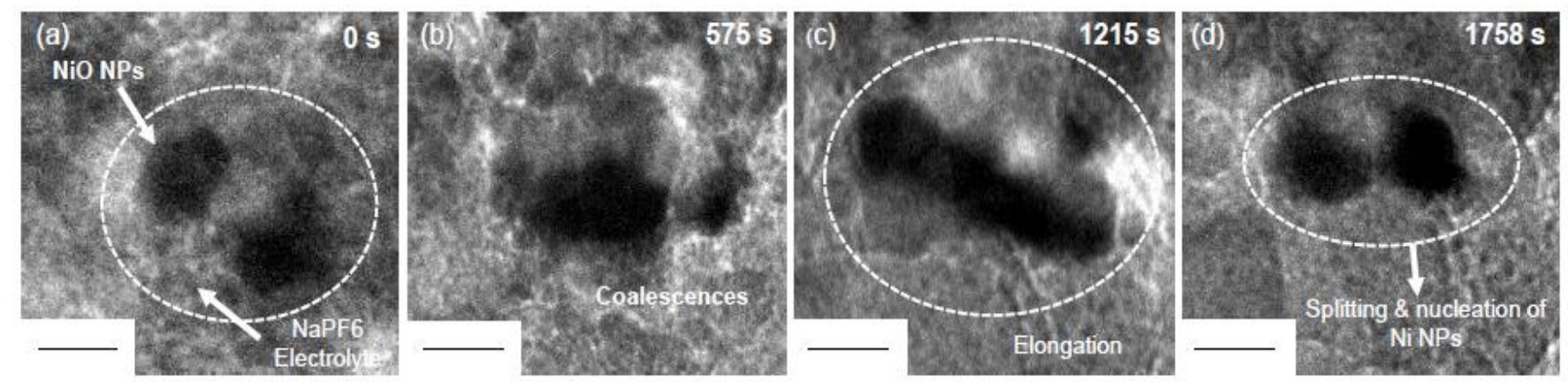

Figure 1. Time series images shows morphological evolutions during an in situ TEM sodiation of $\mathrm{NiO}$ nanoparticle (scale bar $=100 \mathrm{~nm})$.

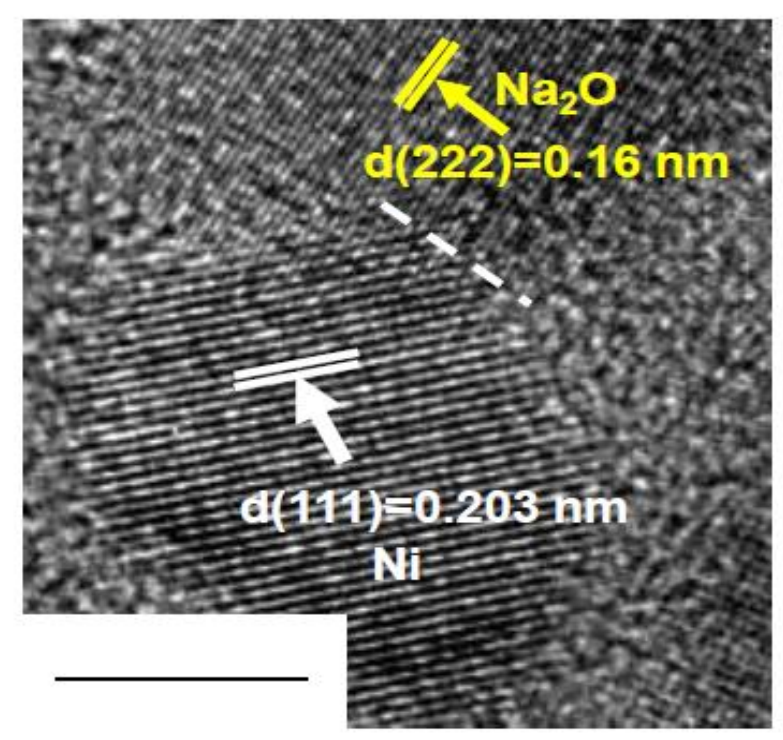

Figure 2. HRTEM image after sodiation, dotted lines shows the interface boundary between $\mathrm{Na}_{2} \mathrm{O}$ and $\mathrm{Ni}$. Lattice fringes for (111) plane of $\mathrm{Ni}$ exhibit incoherent interphase with (222) plane of $\mathrm{Na}_{2} \mathrm{O}$ (scale bar $=20 \mathrm{~nm})$. 